\section{Chromatographic Detection of Free Amino-acids in Normal and Iron-deficient Plants of Hibiscus esculentus L.}

IT has been reported that in iron-deficient plants there is observed an increase of the soluble forms of nitrogen, with a simultaneous decrease of its protein forms ${ }^{1-8}$. This observation was confirmed in the present work, on Hibiscus esculentus L., using the standard methods of chemical analysis. However, we have endeavoured to carry out a qualitative examination of the soluble fraction of nitrogen in normal and iron-deficient plants of this species by the application of paper chromatography.

For that purpose, 3-4 gm. of fresh material taken from 35-day old plants was carefully broken up in 80 per cent ethyl alcohol and the crude extract centrifuged for $15 \mathrm{~min}$. at 4,000 r.p.m. The supernatant liquid was then separated by decantation and concentrated by evaporation until its volume was reduced to 1 c.c. From that concentrated extract 0.005 c.c. was used for each chromatogram. Twodimensional ascending chromatography on Whatman No. 1 paper, $25 \mathrm{~cm} . \times 25 \mathrm{~cm}$. sheets, was adopted, with phenol as solvent for one direction and $n$-butanol/ acetic acid/water $(4: 1: 5)$ for the other. The position of amino-acids was located by spraying with ninhydrin reagent $(0 \cdot 1$ per cent in acetone). The aminoacids were identified by comparing the spots appearing on the chromatograms with those produced by authentic compounds.

In Fig. 1 are shown two chromatograms : $(A)$ from an iron-deficient plant; $(B)$ from a normal plant. With regard to the quantity of amino-acids involved, the greater concentration of these acids is clearly observed in the alcoholic extract of the iron-deficient plants, from the size as well as the intensity of the colour shown by the spots. The higher concentration of asparagine is especially remarkable. From the qualitative point of view, the iron-deficient plants contain phenylalanine, serine and probably glycine. Normal plants do not contain detectable amounts of these amino-acids. From the undetermined spots, two were common on both chromatograms, but were more intense on those prepared from iron-deficient plants. One spot appeared only on the chromatogram from iron-deficient plants, while three appeared on those from normal plants only. The location and colour of one of the last three spots coincide with those produced by cysteinic acid.

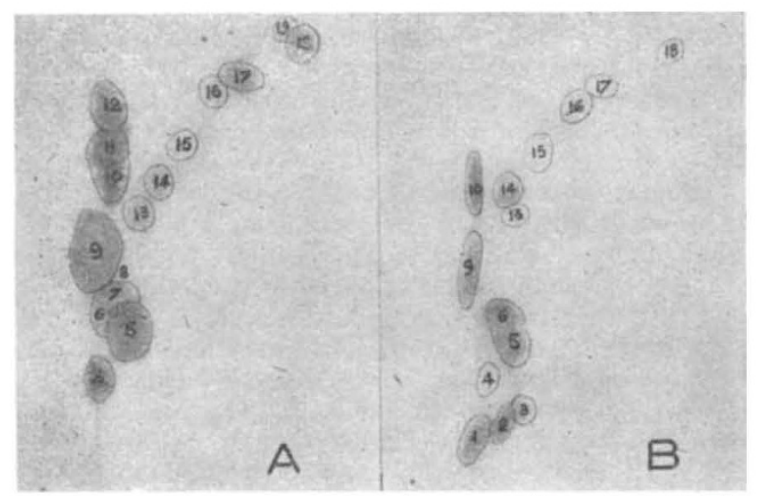

Fig. 1. Two-dimensional ascending paper chromatographs of alcoholic extracts of $\boldsymbol{H}$ ibiscus esculent us $\mathbf{L}$. tissue. $(\boldsymbol{A})$ From an
iron-deficient plant $;(B)$ from a normal plant. $1,2,3,4,12,13$, Unidentified spots ; 5 , aspartic acid ; 6 , glutamic acid ; 7, serine ; 8, glycine (?); 9, asparagine; 10, arginine

11, glutamine; $14, a$-alanine; $15, \beta$-alanine; 16 ,
From these observations, it becomes evident that the difference in the amino-acid content of normal and iron-deficient plants is not only quantitative but also qualitative.

Further details of this work will be published elsewhere.

Laboratory of Non-parasitic Diseases,

Benaki Phytopathological Institute, Kiphissia-Athens. July 3.

${ }^{1}$ Bennett, J. P., Soil Sci., 60, 91 (1945).

${ }^{2}$ Iljin, W. S., Plant and Soil, 3, 239 (1951).

${ }^{3}$ Sideris, C. P., et al., Plant Physiol., 21, 75 (1946).

\section{Chemical Nature of Nucleic Acids in Cyanophyceae}

THE available information regarding the chemical nature of nucleic acids in Cyanophyceae is very meagre, and deoxyribonucleic acid and ribonucleic acid have not been isolated chemically from this group. As the blue-green cell is peculiar in its nuclear structure, the chemical nature of its nucleic acids may be different in kind or degree from that of other sources. With this in view, the nucleic acids were isolated from Nostoc muscorum using the conventional method of Schmidt and Thannhauser as modified by Heinrich et al. ${ }^{1}$.

The deoxyribonucleic acid separated from Nostoc muscorum gives a positive test with the Dische reagent, indicating the presence of deoxypentosesugar. Chromatographic analysis of the hydrolysate according to the procedure of Wyatt ${ }^{2}$ revealed only three spots of bases, corresponding to guanine, adenine and cytosine, but thymine could not be detected. Magee and Burris ${ }^{3}$ showed in experiments on nitrogen fixation by Nostoc muscorum that incorporation of nitrogen-15 in thymine is much less than that of other nitrogenous compounds. It is possible that the thymine in deoxyribonucleic acid is present in too small an amount to be detected on the chromatogram, and hence application of carbon14 as a tracer might help its detection. Carbon-14 dioxide was therefore passed through a Nostoc suspension in phosphate buffer at $p \mathrm{H} 7$ for $6 \mathrm{hr}$. in presence of light. After the experiment, the suspension was extracted with 80 per cent ethanol, alcohol-ether $(3: 1)$ and finally with ether only, and deoxyribonucleic acid was precipitated by the procedure referred to above. An radioautograph revealed the spot corresponding to thymine. Added thymine co-chromatographed with the thymine eluted from the radioactive spot. Thus deoxyribonucleic acid in Cyanophyceae is similar in kind to that from other sources, which also support the earlier observations from cyto-chemical work ${ }^{4}$ and with the electronmicroscope (Singh, R. N., personal communication).

Ribonucleic acid which was precipitated from the deoxyribonucleic acid filtrate by alcohol gave a positive reaction in the orcinol test, indicating the presence of pentose sugar. Chromatographic analysis following the method of Smith and Markham ${ }^{5}$ gave four spots corresponding to guanine, adenine, cytidylic acid and uridylic acid. Thus the ribonucleic acid of Cyanophyceae shows no qualitative deviation from the normal type.

Further studies, with carbon-14 and phosphorus-32, on the turnover of nucleic acids in Cyanophyceae are now in progress. 